American Journal of Infectious Diseases 2 (4): 210-218, 2006

ISSN 1553-6203

(C) 2006 Science Publications

\title{
Consequence on Treatment of TB Patients Affected by HIV/AIDS A Conceptual Research
}

\author{
${ }^{1}$ Sarder Nasir Uddin, ${ }^{1}$ S.J.Hossain, ${ }^{1}$ Md. N. Huda, ${ }^{1}$ M.H. Rahman and ${ }^{2}$ Md Eunus Ali \\ ${ }^{1}$ Biotechnology and Genetic Engineering Discipline, Khulna University, Bangladesh \\ ${ }^{2}$ Pharmacy Discipline, Khulna University, Bangladesh
}

\begin{abstract}
Mycobacterium tuberculosis is an ancient malady, which is one of the world's most wide spread infectious bacterial agents. Fully one-third of the world's population is already infected with Mycobacterium tuberculosis, with the greatest burden of disease and infection borne by people in developing countries. Tuberculosis disease is still out of control. Alarming spread of Human Immunodeficiency Virus (HIV) and emergence of drug resistance is now further complicating the major problem. HIV not only makes the diagnosis of TB more difficult; it contributes to an increase in TB incidence. The rate of breakdown to clinical TB in individuals infected both with HIV and tuberculosis is many times higher than in those without HIV. The present vaccine is not sufficient to reduce the death rate by eradicating TB with HIV/AIDS. The present review is based on the prevention and treatment of TB patient and co-infected with HIV/AIDS and effect of HIV/AIDS on the treatment and prevention of TB. It will help assuming idea about future steps in prevention and treatment of TB among HIV/AIDS patients.
\end{abstract}

Key words: TB, HIV, AIDS, patients

\section{INTRODUCTION}

Tuberculosis is an increasing and major worldwide problem, especially in Africa where AIDS facilitates the spread. It is estimated that nearly 1 billion people will become newly, over 150 million will become sick and 36 million will die worldwide between now and 2020 if control is not further strengthened. Each year there are more than 8.7 million cases and close to 2 million deaths attributed to TB; 100,000 of those 2 million deaths occur among children. In the 1940s, scientists discovered the first of several drugs now used to treat TB. As a result, TB slowly began to disappear in the United States. But TB has come back between 1985 and 1992, the number of TB cases increased. The country became complacent about TB and funding to TB problems was decreased. However, with increased funding and attention to the TB problem, we have had a steady decline in the number of persons with TB. But TB is still a problem; more than 16,000 cases were reported in 2000 in the United States ${ }^{[1,2]}$.

Projections of the future toll of the global TB pandemic are even more frightening. Currently, it is estimated that less than halves of all TB cases worldwide are diagnosed and fewer than 60 percent of diagnosed cases are cured ${ }^{[3]}$ Without unprecedented efforts to improve TB control in regions hardest hit by the disease, incidence is expected to climb steadily ${ }^{[4]}$. Tuberculosis will remain one of the world's top ten causes of adult mortality in the year $2020^{[5]}$.
Since AIDS was first described in the United States, this country has majority of this reported cases and now a day it has been reported from more than 163 countries around the world and the pool of HIVinfected person in Africa and Asia is large and expanding ${ }^{[6]}$. There are some encouraging data which suggests that rapid increase in HIV prevalence have been observed in India, Vietnam, Myanmar (Burma) and South Africa and it is predicted that South Africa will experience one of the worst epidemics in Africa. The World Health Organization (WHO) estimated that, at the end of 1997; 30.6 million people worldwide were living with HIV/AIDS of which over $90 \%$ were in developing countries, two thirds in sub-Saharan Africa $^{[p]}$.

From the United States, the epidemiological data suggest that the adults developing AIDS may be classified into five groups ${ }^{[6]}$.

* Homosexual or bisexual males constitute by far the largest group, accounting for $60 \%$ of the reported cases. This also includes the $5 \%$ who were intravenous drug abuser.

* Intravenous abusers with no previous history of homosexuality compose the next largest group, which represents about $23 \%$ of all the patients. This group represents the majority of all cases among heterosexuals.

* Hemophiliacs, especially those, who received large amount of factor VIII concentrates before 1985, make up $1 \%$ of all cases.

Corresponding Author: Sarder Nasir Uddin, Assistant Professor, Biotechnology and Genetic Engineering Discipline, Khulna University, Bangladesh 
* Recipients of blood and blood components who are not hemophiliacs but who received transfusions of HIV-infected whole or components; for example platelets, plasma account for $2 \%$ of the patients. On the contrary, organs obtained from HIV-infected donors can also transmit AIDS.

* Heterosexuals' contacts members of other high-risk groups (chiefly intravenous drug abusers) constitute $6 \%$ of the patient population.

In approximately $6 \%$ of cases, risk factors cannot be determined. The pediatric population comprises $2 \%$ of all AIDS cases. The remaining $20 \%$ are hemophiliacs and others who have a history of reception of bloods or blood products before $1985^{[6]}$.

At least one in every three HIV-positive adults worldwide is latently infected with the TB bacillus, but not actively sick with TB and not infectious to others. TB-prevalence therapy is aimed at preventing progression from latent disease to active TB. The World Health Organization supports the introduction of TB-preventive therapy, specifically, six months of isoniazid daily for the benefit of people co-infected with HIV and the TB bacillus in countries where national HIV/AIDS programs are able to supply adequate HIV counseling and testing facilities and those that have well-functioning DOTS program ${ }^{[8]}$.

\section{TB and HIV/AIDS}

Evidence for an association between TB and HIV ${ }^{[9]}$ : Studies show that there is a close association between HIV and tuberculosis, one potentiating the impact of the other. The evidence for this interaction includes the following observations:

i. The areas that have been most affected by the HIV epidemic also report the greatest increases in TB cases;

ii. The largest increase in TB cases has occurred among people aged 25-44 years, the age group most affected by AIDS;

iii. $\mathrm{TB}$ is a common opportunistic infection among AIDS patients (between $60 \%-75 \%$ of AIDS patients in the Region will develop TB) and

iv. HIV prevalence among TB patients is higher than that in the general population. For example, rates of up to $40 \%$ have been seen among TB patients compared to $8 \%$ among antenatal clinic attendees in Northern Thailand ${ }^{[10]}$.

Capathogenicity of TB and HIV disease: Human immunodeficiency virus type 1 (HIV-1) and $M$. tuberculosis are two intracellular pathogens that interact at the population, clinical and cellular levels. Initial studies of HIV-1 and TB emphasized the impact of HIV-1 on the natural progression of TB, but mounting immunologic and virologic evidence now indicates that the host immune response to $M$. tuberculosis enhances HIV replication and might accelerate the natural progression of HIV infection ${ }^{[11]}$. Therefore, the interaction between these two pathogens has important implications for the prevention and treatment of TB among HIV-infected persons. Studies of the immune response in persons with TB disease support the biologic plausibility of copathogenesis in dually infected persons. The initial interaction between the host immune system and M. tuberculosis occurs in the alveolar macrophages that present mycobacterial antigens to antigen-specific CD4+ T cells ${ }^{[12]}$. These $\mathrm{T}$ cells release interferon-gamma, a cytokine that acts at the cellular level to activate macrophages and enhance their ability to contain mycobacterial infection. The activated macrophages also release proinflammatory cytokines, such as tumor necrosis factor and interleukin-1, cytokines that enhance viral replication in monocyte cell lines in vitro ${ }^{[13]}$. The mycobacteria and their products also enhance viral replication by inducing nuclear factor kappa-B, the cellular factor that binds to promoter regions of $\mathrm{HIV}^{[14]}$.

When TB disease develops in an HIV-infected person, the prognosis is often poor, though it depends on the person's degree of immunosuppression and response to appropriate antituberculosis therapy $y^{[15]}$. The 1-year mortality rate for treated, HIV-related tuberculosis ranges from $20 \%$ to $35 \%$ and shows little variation between cohorts from industrialized and developing countries ${ }^{[16]}$. The observed mortality rate for HIV-infected persons with TB is approximately four times greater than the rate for TB patients not infected with $\mathrm{HIV}^{[16]}$. Although the cause of death in the initial period of therapy can be $\mathrm{TB}^{[17]}$. Death after the induction phase of anti-tuberculosis therapy usually is attributed to complications of HIV other than $\mathrm{TB}^{[18]}$. Epidemiologic data suggest that active TB accelerates the natural progression of HIV infection. In a retrospective cohort study of HIV-infected women from Zaire, investigators estimated the relative risk of death to be 2.7 among women with active TB compared with those without $\mathrm{TB}^{[19]}$. In a retrospective cohort study of HIV-infected subjects from the United States, active TB was associated with an increased risk for opportunistic infections and death ${ }^{[15]}$. The risk of death, or hazard rate, for persons with HIV-related TB follows a bimodal distribution, peaking within the first 3 months of antituberculosis therapy and then again after 1 year ${ }^{[11]}$; the reasons for this distribution are not clear but might relate to the impact of TB on HIV disease progression. The observation that active TB increases deaths associated with HIV infection has been corroborated in studies of three independent cohorts in Europe $^{[7]}$.

Early in the HIV epidemic, researchers postulated that the immune activation resulting from concurrent infection with parasitic or bacterial pathogens might alter the natural progression of HIV infection ${ }^{[20]}$. Subsequent observations have demonstrated that immune activation from TB enhances both systemic 
and local HIV replication. In some patients with active TB, the plasma HIV RNA level rises substantially before TB is diagnosed ${ }^{[14]}$. Moreover, TB treatment alone leads to reductions in the viral load in these dually infected patients. TB and HIV also interact in the lungs, the site of primary infection with $M$. tuberculosis. In a recently published study of HIVinfected patients with $\mathrm{TB}$, researchers found that the viral load was higher in the bronchoalveolar lavage fluid from the affected versus the unaffected lung and was correlated with levels of tumor necrosis factor in bronchoalveolar fluid ${ }^{[21]}$. Researchers used V3 loop viral sequences to construct a phylogenetic tree and observed that the HIV quasispecies from the affected lung differed from those in the plasma within the same patient. These data suggest that pulmonary TB might act as a potent stimulus for the cellular-level replication of HIV. In summary, recent research findings have improved clinicians' understanding of how HIV affects the natural progression of TB and how TB affects the clinical course of HIV disease and these findings support the recommendation for prevention, early recognition and effective treatment for both diseases.

HIV and TB alter each other pathogenesis and natural course: The pathogenesis of both tuberculosis infection and the disease relates directly to cellmediated immunity (CMI). Especially CD4+ Tlymphocytes. Not surprisingly, HIV infection which induces CD4+ T-lymphocytes depletion, also leads to defective immunological response to $M$. tuberculosis. the pathogenesis of TB can be altered by HIV either through reactivation of latent tuberculosis infection to active disease (more common) or by causing rapid progression from recent infection with $\mathrm{M}$. tuberculosis to tuberculosis disease ${ }^{[10]}$. Findings from several studies suggest that active TB may accelerate HIVinduced immunological deterioration. The evidence is as follows:

* Active TB is associated with transient CD4+ Tlymphocyte depletion;

* TB cause immune stimulation and increased production of cytokines, such as tumour necrosis factor (TNF) which increases HIV replication in vitro and

* HIV-infected persons with TB appear to have a higher risk of opportunistic infections and death than do HIV-infected patients with similar CD4+ $\mathrm{T}$-lymphocyte counts but without $\mathrm{TB}^{[10]}$.

The clinical features of TB more commonly seen in HIV-positive individuals:

* As HIV infection progresses, CD4+ lymphocytes decline in number and function. Therefore, the immune system is less able to prevent the growth and local spread of M. tuberculosis. As a result, disseminated and extra-pulmonary disease is more commonly seen. Nevertheless, pulmonary TB is still the most common from of TB even in HIVinfected patients. Many studies reveal that pulmonary involvement occurs in $70-90 \%$ of all patients with TB.

Pulmonary TB: The presentation of pulmonary TB depends on the degree of immune suppression. The Table 1 shows how the clinical features, results of sputum smears and chest X-ray appearance differs in early and late HIV infection. In advanced HIV infection, the presence of many opportunistic infectious affecting the lungs may cause difficulties in the diagnosis of TB. The occurrence of hilar and mediastinal adenopathy by chest X-ray can also suggest the diagnosis of TB in an HIV-infected patient ${ }^{[10]}$.

Extra-pulmonary TB: The most common forms of extra-pulmonary involvement include lymphadenopathy, pleural effusion, pericardial disease, military disease and meningitis, cervical, supraclavicular and auxiliary lymphnodes are the most common sites of peripheral lymphadenitis. Percutanaous needle aspiration of the lymph nodes can be useful in establishing the diagnosis ${ }^{[10]}$.

HIV infection influences the diagnosis of TB: In most cases, the clinical presentation of tuberculosis among patients with HIV is indistinguishable from other cases. However, some may show a bizarre pattern with a higher proportion of cases tending to have a negative sputum smear. In spite of that, sputum smear examination remains an essential component in the diagnosis of tuberculosis in countries where HIV infection is common because of its ability to identify infectious cases.

The diagnosis of TB in HIV-positive patients is more difficult for three main reasons:

* Proportion of HIV-positive patients with pulmonary TB will have positive sputum smears;

* X-ray abnormalities, which are not specific for TB in HIV-negative patients, are even more nonspecific in HIV-infected with only minor abnormalities on chest X-ray or with abnormalities which do not look like classical TB and

* Patients infected with have frequent illnesses with pulmonary involvement caused by agents other than M. tuberculosis ${ }^{10}$.

Treatment of TB patients affected by HIV/AIDS: The response to anti-TB therapy is the same for HIVpositive and HIV-negative TB patients. The only exception is that thiacetazone is not recommended for HIV-positive TB patients because it may increase the risk of severe and sometimes fatal skin reactions;

Short-course treatment hastens cure and prolongs survival in HIV-infected persons ${ }^{[22]}$.

HIV-related immuno-suppression does not interfere with the effectiveness of therapy for TB. Since the management of TB and the response to standard short course regimen is similar in patients with or without HIV, there is no rationale for HIV tests in the clinical settings from the patient management of view. TB bacteria die very slowly. 
(range from 60\%-90\%). However, it is still not clear as to how preventive therapy must be given ${ }^{[10]}$.

In Malawi, smear-negative pulmonary tuberculosis and extra-pulmonary tuberculosis are more common in TB patients with HIV infection; $75 \%$ of TB patients are HIV-positive.

Treatment outcomes depend on the HIV status, type of TB and age group. HIV-infected patients had a higher death rate than HIV-negative TB patients $(60 \%$ vs $6 \%$ ) and one third of all HIV-positive patients and half of the new HIV-positive smear-negative pulmonary patients died during treatment. The reason for high death rates in smear-negative patients is probably due to incorrect diagnosis, as three are many operational difficulties in resource-poor settings in Sub-Saharan Africa $^{[23]}$.

247 consecutive HIV-infective patients with smearpositive pulmonary or clinically confirmed extrapulmonary tuberculosis were compared with 312 HIV-negative TB patients. Mortality during treatment was higher in HIV-infected patients ( $6 \%$ vs $0.4 \%)$ and was even higher $(10 \%)$ in HIV-infected patients with low $(<200 / \mu \mathrm{L}) \mathrm{CD} 4+$ lymphocyte counts ${ }^{[24]}$.

Tuberculosis and HIV can be effectively treated concomitantly, as long as appropriate dose adjustments are made to account for drug-drug interactions. Treatment of both diseases simultaneously appears to result in a substantial improvement in morbidity and mortality compared to treatment of tuberculosis alone.

Persons with HIV infection and TB usually can be treated with standard anti-TB regimens with good results, although in some cases, prolonged therapy may be warranted. Patients with TB and HIV may also have more rapid resolution of their TB if the HIV infection is treated concurrently ${ }^{[25]}$. Therefore, it is important for clinicians to be aware of the HIV status of their patients with TB. Testing of persons with TB for infection with HIV is an important intervention point for counseling and testing for HIV infection and for administration of antiretroviral therapy and prophylaxis for opportunistic infections. Since treatment of HIV may require protease inhibitors or nonnucleoside reverse transcriptase inhibitors and TB therapy for persons receiving these drugs may preclude the use of rifampin, this may lead to alteration of the anti-TB regimen ${ }^{[8]}$.

In the United States, $14 \%$ of persons with TB in 1993-1994 also were reported to have AIDS and in some areas, HIV infection is seen in as many as $58 \%$ of patients with $\mathrm{TB}^{[26]}$. While the rate of co infection varies widely among different geographic areas, clinicians are poor predictors of which patients are likely to have HIV infection. Therefore, HIV testing is recommended for and should be offered to all patients with newly diagnosed TB. However, because not all patients will accept HIV serologic testing, $80 \%$ rather than $100 \%$ is the proposed performance indicator ${ }^{[27]}$.

HIV-infected patients who received directly observed, intermittent treatment were more likely to complete treatment than were HIV infected patients who received short-course chemotherapy without directly observed treatment. HIV infected patients who received directly observed treatment were also much likely to survive ( $85 \%$ vs $57 \%, \mathrm{p}=0.01)$. This improved survival was found in multivariate as well as univariate analysis $^{[28]}$.

The HIV epidemic caused an increase in the notification rates of smear-positive tuberculosis in adults. The study indicates that the presence of an effective DOTS programme curtailed tuberculosis transmission despite a substantial increase in TB due to the HIV epidemic ${ }^{[29]}$.

A study found that DOTS can prevent drugresistant TB in the context of a HIV epidemic in lowincome counties, but could not prevent the increase in incidence of TB. This increase is likely due primarily to reactivation of latent tuberculosis infection in HIVinfected persons in this area where the prevalence of HIV in the adult population exceeds $20 \%{ }^{[27]}$.

Directly observed treatment, short course (DOTS): DOTS as the International Union against TB and Lung Disease (IUTLD) pioneered a TB control strategy. The DOTS strategy takes simple technology-the successful components of TB control and packages it with good management practices for widespread use. The strategy can be easily integrated into general health services and can therefore be widely used. How the DOTS strategy is implemented varies from country to country and within countries. The implementation of DOTS requires flexibility in adapting the strategy according to local circumstances. There are five key elements that are considered essential for the implementation of the DOTS strategy ${ }^{[10]}$.

The principles of DOTS were first developed in the national TB programme in Tanzania and subsequently expanded to a further six countries in Africa and to Nicaragua, with the assistance of IUTLD. The brand name "DOTS" was born in 1994. Modifying the commonly used DOT acronym to include another key element of he strategy-the Short-course form "SCC"now gave meaning to "DOTS" $[10]$.

The five major components of DOTS ${ }^{[10]}$ :

i. Political commitment and resources: TB control is a public health responsibility and top-down support is crucial. This component must be the strongest link in the chain. 
ii. Microscopy: Accurate diagnosis using sputumsmear microscopy among symptomatic patients is the first step in early detection of active TB infection. It sets the DOTS cure cycle in motion and protects others from infection.

iii. Treatment: Standardized six-to eight-month regimens for all patients with active TB, with Directly observed treatment for at least the first two months, is necessary. The Success of this phase is contingent upon a sound, functional health-sector infrastructure and trained personnel.

iv. Medicines: Regular, uninterrupted supplies of the four to six most effective anti-TB drugs are essential. Full compliance with the drug regimen results in nine out of ten patients being cured.

v. Monitoring: A standardized recording and reporting system allows assessment of each patient's treatment and progress. Rigorous overall record-keeping also acts as early warning for emerging disease trends (such as MDR-TB) $)^{[10]}$.

Specialty about DOTS: DOTS cure active TB. It is remarkably effective. Without treatment, seven in ten people with infectious TB will die of he disease, on average within four to five years of onset, even if they are young when they contract the disease ${ }^{[30]}$. Though non-DOTS considerably, such programs are usually less successful at curing TB. Many sufferers remain chronically ill and continue to unknowingly transmit the disease to family, friends and even strangers.

Conversely, good DOTS programs rapidly reduce both death and disease, curing more than 85 percent of patients. In human terms, DOTS gives young people marked for premature TB death a chance to lead full and productive lives, raise children to adulthood and make contributions to their communities and society. Additionally:

i. DOTS saves lives: TB deaths during the period 20012005, in addition to the 4.2 million lives saved through ongoing DOTS expansion program. Even today, in China alone DOTS Modeling suggests that achievement of WHO's 2005 target would avert 15.5 million has prevented 46 percent of deaths that would otherwise have occurred in the provinces in which the program is being applied. This translates into 30,000 lives saved each year ${ }^{[23]}$.

ii. DOTS stops the chain reaction of transmission: Curing people with TB prevents them from infecting others. For example, introducing DOTS in Peru have accelerated the decline in notified TB incidence to about 7 percent per year ${ }^{[31]}$.

iii. DOTS prevents treatment failure and the emergence of even more deadly strains of drugresistant TB: For example, the China Tuberculosis Coalition (CTC) reported that the failure rate in Previously treated patients fell from 17.6 percent to 6.2 percent following the Introduction of DOTS in World Bank-assisted provinces in China.

iv. DOTS reduces TB recurrence rate: For example, in the U.S. state of Texas, TB recurrence rates fell from 20.9 percent to 5.5 percent within six years when a DOTS-based TB-control strategy was introduced ${ }^{[32]}$.

v. DOTS indirectly alleviates poverty: Saving lives, reducing periods of illness and prevention of new infections means Fewer years of productive work lost.

vi. DOTS overcome TB's stigma: Effective treatment, combined with a positive approach, reduces the fear of death and disability that has fuelled the profound stigma often associated with TB. In Nepal, for example, the introduction of DOTS has led to a general awareness that TB is curable. As a result, TB is now less feared, no longer "Khapate"-the disease that "dries you up" before you die.

vii. DOTS provides a model for strengthening health services: Remarkably successful in promoting the development of peripheral health services, the DOTS strategy can serve as a model for expanded use of HIV antiretrovirals, as proposed in Malawi ${ }^{[33]}$. If adaptations of the DOTS strategy were shown to be effective in AIDS treatment, networks linked to DOTS TBtreatment programmed could rapidly be set up, given that up to one-third of all AIDS patients die of tuberculosis.

viii. DOTS save taxpayer's money and lives: The World Bank has hailed DOTS as "one of he most costeffective interventions available". Country studies in the early 1990s from Malawi, Mozambique and Tanzania showed the cost of TB interventions ranging from \$19-52 per life saved. But drugs cost up to four times as much at that time. Today, the DOTS drug package can be purchased for as little as $\$ 10^{[34]}$. This means that investing in TB control will save lives, immediately. Over time, TB control will also "turn a profit" as it reduces the disease burden on society.

BCG vaccination: A Bacillus Calmette Guerin (BCG) is a live vaccine derived from an attenuated strain of $\mathrm{M}$. 
bovis and is named after the two doctors Calmette and Guerin (1920) who discovered it. BCG is effective in preventing disseminated form of $\mathrm{TB}$ in children; efficacy is debatable against adult forms of TB. It is a major component of global TB-control efforts.

BCG cannot be expected to prevent TB in HIVpositive individuals since young adults usually acquire HIV infection. As BCG is mainly given at birth in developing countries, the immunity from it wanes by the age of young adult; BCG cannot against the exogenous re-infection of TB adults.

BCG is not safe in individuals with AIDS because of the risk of disseminated BCG disease. Presently, WHO and UNICEF recommended that, for a symptomatic HIV-infected children living in areas where the risk of tuberculosis is high, BCG still be given at birth or as soon as possible thereafter in accordance with standard childhood immunization policies, but that it be withheld in infants thought to have symptomatic HIV-infection.

The effectiveness of BCG in general, particularly in developing countries where it is given at birth or less than one year of age, is limited to protection against the severe forms of TB in children. The degree of effectiveness varies between 0 and $80 \%$ but the vaccine is unable to prevent most contagious forms of TB in adults.

Various approaches are being taken to prevent infection and also, more speculatively, to prevent reactivation of patients already infected. These approaches include DNA vaccines, recombinant BCG and other live vectors, attenuated M. tuberculosis, lipid antigens and relatively simple secreted antigens ${ }^{[34]}$.

Adverse reactions are generally more common in HIV-positive than in HIV-negative TB patients. Most reactions occur in the first two months of treatment. Skin rash and hepatitis are more common and most often attributed to rifampicin. The usual drug responsible for fatal skin reaction such as exfoliative dermatitis, Steven-Johnson syndrome and toxic epidermal necrolysis is thiacetazone. Therefore, thiacetazone should never be given to HIV-positive TB patients. From a programmatic point of view, thiacetazone should not be prescribed in areas where HIV prevalence is shown to be high ${ }^{[10]}$.

\section{CONCLUSION}

Autopsy studies have shown that TB accounts for up to one-third of AIDS deaths ${ }^{[35]}$. Controlling TB in high HIV prevalence areas will require, first of all, a highly effective DOTS program. The DOTS expansion plans include cost estimates for building and sustaining successful programs in all high-burden countries, including countries with high HIV-infection rates. Once DOTS programs are well established in high HIV- prevalence countries, they will likely need to be supplemented with active case-finding initiatives for co-infected patients. The strategy has been successfully demonstrated also among HIV-positive TB patients, with increased completeness of therapy and improved patient survival ${ }^{[8]}$. All HIV-infected persons at risk for infection with $M$. tuberculosis must be carefully evaluated and if indicated, administered therapy to prevent the progression of latent infection to active TB disease and avoid the complications associated with HIV-related TB. All HIV-infected patients undergoing treatment for TB should be evaluated for antiretroviral therapy, because most patients with HIV-related TB are candidates for concurrent administration of antituberculosis and antiretroviral drug therapies. However, the use of rifampin with protease inhibitors or non-nucleoside reverse transcriptase inhibitors is contraindicated $^{[36]}$.

Ideally, the management of TB among HIVinfected patients taking antiretroviral drugs requires a) directly observed therapy, b) availability of experienced and coordinated $\mathrm{TB} / \mathrm{HIV}$ care givers and in most situations, c) use of a TB treatment regimen that includes rifabutin instead of rifampin. Because alternatives to the use of rifampin for antituberculosis treatment are now available, the previously recommended practice of stopping protease inhibitor therapy to allow the use of rifampin for TB treatment is no longer recommended for patients with HIV-related TB. The use of rifabutin-containing antituberculosis regimens should always include an assessment of the patient's response to treatment to decide the appropriate duration of therapy (i.e., 6 months or 9 months). Physicians and patients also should be aware that paradoxical reactions might occur during the course of TB treatment when antiretroviral therapy restores immune function ${ }^{[37]}$.

One hundred eighteen years after Koch's discovery of the tuberculosis bacterium and 80 years after the development of the BCG vaccine, the tuberculosis pandemic is still out of control. The development of new, stable, possibly DNA-based TB vaccine is the hope of tomorrow, but the researchers involved made it completely clear that we are still far from achieving this. Hygienic measures and carefully monitored follow-up and treatment (Directly Observed Treatment, DOT) of TB cases is cost and environmentally effective even today ${ }^{[38]}$. According to Hong Kong Chest Service/Medical Research Council, (1976) the tuberculosis has some side effects, which should be considered to reduce as much as possible. This strategy is now spreading with WHO help to many poor countries. Future research can be including:

* Epidemiological research to assess the extent of the spread of TB to HIV- negative \& HIV- positive people. 
* The development of methods for early and accurate diagnosis of $M$. tuberculosis infection in persons coinfected with HIV.

* Basic research to define what host factors protect persons from infection with $M$. tuberculosis and HIV and from the development of TB and HIV disease.

\section{REFERENCES}

1. Ackah, A.N., D. Coulibaly and H. Digbeu, 1995. Lancet, 345: 607-610.

2. Garrett, 2000. Of epidemic proportions/UN report: AIDS deaths to surpass plague, Spanish flu. Newsday, 29 November A08.

3. Raviglione, M.C., C. Dye, S. Schmidt and A. Kochi, 1997. Assessment of worldwide tuberculosis control. WHO Global Surveillance and Monitoring Project. Lancet, 350:624-9; World Health Organization. Tuberculosis and Sustainable Development: Report from the Ministerial Conference in Amsterdam. Geneva: World Health Organization, 2000. WHO/CDS/STB/2000.6.

4. Dye, C., G.P. Garnett, K. Sleeman and B.G. Williams, 1998. Prospects for worldwide tuberculosis control under the WHO DOTS strategy. Directly observed short-course therapy. Lancet, 352:1886-91; Murray CJL; Styblo K, and Rouillon A. tuberculosis. In: Jamison.

5. Murray, C.J. and A.D. Lopez, 1997. Alternative projections of mortality and disability by cause 1990-2020: Global Burden of Disease Study. Lancet, 349: 1498-504.

6. Cotran, R.S., V. Kumar and S.L. Robbins, 1994. Pathological Basis of Disease. $5^{\text {th }}$ Edn. Eastern Press Pvt. Ltd., Bangalore-560029.

7. Grant, A.D. and K.M. DeCock, 1998. The growing challenge of HIV/AIDS in developing countries. Br. Med. Bull., 54: 369-381.

8. Becerra, M.C., J. Freeman and J. Bayona, 2000. Using treatment failure under effective directly observed short-course chemotherapy programs to identify patients with multidrug-resistant tuberculosis. Int. J. Tuberc. Lung Dis., 4: 108-14.

9. Whalen, C., C.R.Jr. Horsburgh, D. Hom, C. Lahart, M. Simberkoff and J. Ellner, 1997. Site of disease and opportunistic infection predict survival in HIVassociated tuberculosis. AIDS, 11: 455-60.

10. Rojanapithayakorn, W. and J.P. Narain, 1999. Tuberculosis and HIV: World Health Organization, Regional Office For South-East Asia, New Delhi, India, ISBN 974-415-081-5.
11. Whalen, C., A. Okwera and J. Johnson, 1996. Predictors of survival in human immunodeficiency virus-infected patients with pulmonary tuberculosis. Am. J. Respir. Crit. Care Med., 153: 1977-81.

12. Toossi, Z., 1996. Cytokine circuits in tuberculosis. Infectious Agents and Disease, 5: 98-107.

13. Duh, E.J., W.J. Maury, T.M. Folks, A.s. Fauci and A.B. Rabson, 1989. Tumor necrosis factor alpha activates human immunodeficiency virus type 1 through induction of nuclear factor binding to the NF-kappaB sites in the long terminal repeat. Proc. Natl. Acad. Sci., USA, 86: 5974-8.

14. Goletti, D., D. Weissman and R.W. Jackson, 1996. Effect of Mycobacterium tuberculosis on HIV replication. Role of immune activation. J. Immunol., 157: 1271-8.

15. Whalen, C., C.R. Horsburgh, D. Hom, C. Lahart, M. Simberkoff and J. Ellner, 1995. Accelerated course of human immunodeficiency virus infection after tuberculosis. Am. J. Respir. Crit. Care Med., 151: 129-35.

16. Perriens, J.H., R.L. Colebunders and C. Karahunga, 1991. Increased mortality and tuberculosis treatment failure rate among human immunodeficiency virus (HIV) seropositive compared with HIV seronegative patients with pulmonary tuberculosis treated with "standard" chemotherapy in Kinshasa, Zaire. Am. Rev. Respir. Dis., 144: 750-5.

17. Nunn, P., R. Brindle and L. Carpenter, 1992. Cohort study of human immunodeficiency virus infection in patients with tuberculosis in Nairobi, Kenya. Am. Rev. Respir. Dis., 146: 849-54.

18. Small, P.M., G.F. Schecter, P.C. Goodman, M.A. Sande, R.E. Chaisson and P.C. Hopewell, 1991. Treatment of tuberculosis in patients with advanced human immunodeficiency virus infection. N. Engl. J. Med., 324: 289-94.

19. Braun, M.M., N. Badi and R.N. Ryder, 1991. A retrospective cohort study of the risk of tuberculosis among women of childbearing age with HIV infection in Zaire. Am. Rev. Respir. Dis., 143: 501-4.

20. Quinn, T.C., P. Piot and J.B. McCormick, 1987. Serologic and immunologic studies in patients with AIDS in North America and Africa. The potential role of infectious agents as cofactors in human immunodeficiency virus infection. JAMA, 257: 2617-21.

21. Nakata, K., W.N. Rom and Y. Honda, 1997. Mycobacterium tuberculosis enhances human immunodeficiency virus-1 replication in the lung. Am. J. Respir. Crit. Care Med., 155: 996-1003. 
22. Odwera, A., C. Whalen and F. Byekwaso, 1994. Lancet, 344: 1323-1328.

23. Dye, C.L., Z. Fengzeng, S. Scheele and B. Williams, 2000. Evaluating the impact of tuberculosis control.

25. Centre for Disease Control and Prevention. Prevention and Treatment of tuberculosis among patients infected with human immunodeficiency virus: principles of therapy and revised recommendation. MMWR Morb Mortal Wkly Rep 1998; 47 (RR-20): 1-5. First citation in article.

26. Moore, M. and Mc Cray, 1997. Onorato I. The proportion of U.S. TB cases with a match in the AIDS registry (Abstract). Am. J. Respir. Crit. Care Med., 155: A 23. First citation in article.

27. Folks, T.M., J. Justement, A. Kinter and C.A. Dinarello, 1987. Fauci AS. Cytokine-induced expression of HIV-1 in a chronically infected promonocyte cell line. Science, $238: 800-2$.

28. Alwood, K., J. Keruly, K. Moore-Rice, D. Stanton and R.E. Chaisson, 1994. AIDS, 8: 1103-1108.

29. Tanzania Tuberculin Survey Collaboration. International Journal of Tuberculosis and Lung Disease 2001; 5: 103-112.

30. Dye, C., S. Scheeles, P. Dolin, V. Pathania and M.C. Raviglione, 1999. Global burden of tuberculosis: Estimated incidence, prevalence and mortality by country. JAMA, 282: 677-686.

31. World Health Organization. Global Tuberculosis Control. WHO Report 2001. Geneva.

32. Weis, S.E., P.C. Slocum and F.X. Blais, 1994. The effect of directly observed therapy on the rates of drug resistance and relapse in tuberculosis. N. Engl. J. Med., 330: 1179-84.
33. Farmer, P.E., D.A. Walton and J.J. Furin, 2000. The changing face of AIDS: implications for policy and practice. In: Mayer KH, Pizer H, American Public Health Association; The Emergence of AIDS: The Impact on Immunology, Microbiology, and Public Health. Washington: American Public Health Association.

34. World Health Organization. $105^{\text {th }}$ Session of the Executive Board of the World Health Organization. Stop TB Initiative. Report by the Director General. Geneva: World Health.

35. Lucas, S.B., A. Hounnou and C. Peacock, 1993. The mortality and pathology of HIV infection in a West African city. AIDS, 7: 1569-1579.

36. Okwera, A., C. Whalen and F. Byekwaso, 1994. Randomised trial of thiacetazone and rifampicincontaining regimens for pulmonary tuberculosis in HIV-infected Ugandans. Lancet, 344: 1323-8.

37. Awood, K., J. Keruly, K. Moore-Rice, D. Stanton, C.P. Chaulk and R.E. Chaisson, Effectiveness of supervised, intermittent therapy for tuberculosis in HIV-infected patients.

38. Sven, B., K. Gunilla and A. Jan, 2000. Prevention and Treatment of Tuberculosis in the Coming Century (NS 144), 2000. Nobel Forum, Karolinska Institute, Stockholm Organizers. 